\title{
STATISTISCHER BEITRAG
}

zur

\section{Kenntniss des Vorkommens von Nematoden}

\author{
in Vögeln
}

von

Walter VOLZ.

Arbeit aus der zoologischen Anstalt der Universität Basel

Im zoologischen Institut wurden von Herrn K. WoLfFHüGEL eine grosse Anzahl von Vögeln auf Darmparasiten untersucht. Die Nematoden hat mir derselbe in verdankenswerter Weise zur Bestimmung überlassen; die übrigen Würmer werden von anderer Seite untersucht. Herrn Prof. Dr. F. Zscнокке, der mich bei dieser Arbeit mit Rat und Tat unterstützte, spreche ich hiermit meinen Dank aus. Die Arbeit beschäftigte mich von Mitte Dezember 1897 bis Mitte März 1898. Ich werde mich begnügen, die Resultate dazu zu verwenden, eine Uebersicht über das Vorkommen und die Verbreitung der gefundenen Nematoden zu geben.

In den Zusammenstellungen werden nur die vollkommen sicher bestimmten Arten aufgeführt. Es fanden sich noch ca. 6 Arten, deren Bestimmung einstweilen unmöglich war, letztere stammen aus folgenden Wirten: Corvus cornix, Corvus corone, Garrulus glandarius, Upupa epops, Chunga Burmeisteri, Ardea

Rev. Suisse de Zool., T. 6. 1898. 
cinerea, Gallinula chloropus. Ich behalte mir vor, eventuell später darauf zurückzukommen und die darunter sich findenden neuen Species zu beschreiben.

Wo keine spezielle Bemerkung beigefügt ist, stammt der betreffende Vogel aus der näheren Umgebung von Basel oder Freiburg i. B.

Es wurde fast ausschliesslich der Verdauungstractus auf Parasiten untersucht.

Folgende Tabelle mag zur Orientierung über die gefundenen und bestimmten Nematoden dienen :

\begin{tabular}{|c|c|c|}
\hline Parasit & Wirt & Bewohntes Organ \\
\hline $\begin{array}{l}\text { 1. Ascaris spiralis } \\
\text { Rud. }\end{array}$ & Otus vulgaris. & Intestinum. \\
\hline $\begin{array}{l}\text { 2. Ascaris depressa } \\
\text { Rud. }\end{array}$ & $\begin{array}{l}\text { Astur palumbarius, Accipiter ni- } \\
\text { sus, Buteo vulgaris, Circus } \\
\text { cyaneus. }\end{array}$ & $\begin{array}{l}\text { Intestinum, Ventri- } \\
\text { culus. }\end{array}$ \\
\hline $\begin{array}{l}\text { 3. Ascaris spiculi- } \\
\text { gera Rud. }\end{array}$ & Podiceps minor. & \\
\hline $\begin{array}{l}\text { 4. Ascaris ensicau- } \\
\text { data Rud. }\end{array}$ & $\begin{array}{l}\text { Corvus corone, Corvus frugile- } \\
\text { gus, Garrulus glandarius, } \\
\text { Alauda spec., Turdus viscivo- } \\
\text { rus. }\end{array}$ & $\begin{array}{c}\text { Intestinum, Cavum } \\
\text { abdominale (?) }\end{array}$ \\
\hline $\begin{array}{l}\text { 5. Heterakis inflexa } \\
\text { Rud. }\end{array}$ & $\begin{array}{c}\text { Tetrao urogallus, Tetrao tetrix, } \\
\text { Tetrao bonasius (Russland). }\end{array}$ & Intestinum, Cœсum, \\
\hline $\begin{array}{l}\text { 6. Heterakis vesicu- } \\
\text { laris Fröl. }\end{array}$ & $\begin{array}{l}\text { Tetrao urogallus, Perdix saxa- } \\
\text { tilis, Starna cinerea, Gallus } \\
\text { domesticus, Phasianus colchi- } \\
\text { cus, Phasianus pictus, Cros- } \\
\text { soptilon mantschuricus (in Ge- } \\
\text { fangenschaft), Polyplectronbi- } \\
\text { calcaratum (in Gefangensch.), } \\
\text { Pavo cristatus. }\end{array}$ & Intestinum, Cœcum. \\
\hline $\begin{array}{l}\text { 7. Strongyluspapil- } \\
\text { latus v. Linst. }\end{array}$ & Tetrao tetrix. & Cœеcum. \\
\hline $\begin{array}{l}\text { 8. Strongylus dimi- } \\
\text { diatus Dies. }\end{array}$ & $\begin{array}{l}\text { Rhea americana (zoologischer } \\
\text { Garten). }\end{array}$ & \\
\hline
\end{tabular}


9. Trichosoma Picorum M. C. V.

10. Trichosomaresectum Duj.

11. Trichosoma longicolle Rud.

12. Trichosoma brevicolle Rud.

13. Trichosoma obtusum Rud.

14. Trichosoma Falconum Rud.

15. Trichosoma contortum Crepl.

16. Filaria tricuspis Fedtsch.

17. Filaria horrida Dies.

18. Filaria leptoptera Rud.

19. Filariaattenuata Rud.

20. Dispharagus anthuris Rud.

21. Dispharagus laticeps Rud.

22. Spiroptera truncata Crepl.

23. Physaloptera alata Rud.

24. Angiostomum sanguinolentum (?) v. Linst .
Dryocopus Martius.

Pyrrhocorax alpinus (Schweiz), Lycos monedula, Corvus corone, Corvus frugilegus, Garrulus glandarius, Pica caudata.

Tetrao urogallus, Starna cinerea, Phasianus colchicus.

Otis tarda (zool. Garten).

Otus vulgaris, Brachyotus palustris.

Accipiter nisus.

Corvus corone, Corvus cornix.

Corvus corone, Corvus cornix, Corvus frugilegus, Merula vulgaris.

Rhea americana (zoologischer Garten).

Accipiter nisus.

Garrulus glandarius.

Corvus corone, Corvus cornix, Corvus frugilegus, Garrulus glandarius.

Accipiter nisus.

\section{Upupa epops.}

Accipiter nisus.

Otus vulgaris.
Intestinum .

Intestinum, Cœcum.

Intestinum .

Intestinum.

Oesophagus .

Cavum abdominale.

Cavum thoracale $u$. Musculat. d. hintern Extremität.

Ventriculus, Intestinum.

Cavum abdominale.

Unter der Tunica ventriculi.

Oesophagus, Ingluvies, Ventriculus.

Cavum abdominale. 


\section{TABELLE}

über die Nematodenfauna jedes einzelnen Wirtes.

I. Rhea americhana Lam. (zool. Garten).

Strongylus dimidiatus Dies. $\sigma^{x}$ und $\bigcirc$ in zahlreichen Exemplaren.

Filaria horrida Dies. $\bigcirc^{\top}$ und $\bigcirc$ in zahlreichen Exemplaren.

\section{Astur palumbarius L.}

Ascaris depressa Rud.

\section{AcCipiter Nisus L.}

Von 16 untersuchten Individuen waren 12 mit Nematoden besetzt. Ascaris depressa Rud. bewohnte 3, Trichosoma Falconum Rud. ebenfalls 3, Filaria leptoptera Rud. 4, Dispharagus laticeps Rud. 1, Physaloptera alata Rud. 5 Individuen.

Maximalzahlen : Ascaris depressa 7 ( $1 \mathrm{O}^{7}, 6$ ○), Trichosoma Falcomum 3, Filaria leptoptera 1, Dispharagus laticeps 1, Physaloptera alata 9 (2 ○', 7 ૧).

1 Accipiter nisus enthielt 2 Species (Ascaris depressa und Dispharagus laticeps). 2 enthielten je 3 Species (Trichosoma Falconum, Filaria leptoptera, Physaloptera alata; Ascaris depressa, Filaria leptoptera, Physaloptera alata).

\section{Buteo vulgaris Bechst.}

Untersucht wurden 16 Stück, von diesen enthielten 5 Rundwürmer und zwar nur Ascaris depressa Rud., im Maximum 5-7 Stück.

\section{Circus cyaneus L.}

Ascaris depressa Rud. (1 $\bigcirc^{x}, 1$ \&). 
VI. Otus vulgaris Flemm.

Ascaris spiralis Rud.

Trichosoma obtusum Rud.

Angiostomum sanguinolentum (?) v. Linst.

VII. Brachyotus palustris Forst.

Trichosoma obtusum Rud.

VIII. Pyrrhocorax alpinus L. (Schweiz).

Trichosoma resectum Duj.

IX. Lycos Monedula L.

Trichosoma resectum Duj.

X. Corvus corone L.

Im ganzen wurden 155 Stück untersucht, davon waren 67 frei von Nematoden.

Von Ascaris ensicaudata hud. waren 5, von Trichosoma resectum Duj. 7, von Filaria tricuspis Fedtsch. 5, von Dispharagus anthuris Rud. 77 Exemplare bewohnt; das einzige, dessen Oesophagus untersucht wurde, enthielt von Trichosoma contortum Crepl. 10 @.

Maximalzahlen : Ascaris ensicaudata 1, Trichosoma resectum ca. 40 ( $\sigma^{\top}$ und ?), Trichosoma contortum 10 \&, Filaria

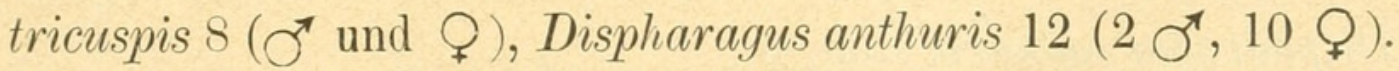

12 Individuen von Corvus corone beherbergten 2 Arten von Nematoden gleichzeitig (Trichosoma resectum, Dispharagus anthuris; Filaria tricuspis, Dispharagus anthuris; Ascaris ensicaudata, Dispharagus anthuris; Trichosoma contortum, Dispharagus anthuris).

\section{Corvus cornix L.}

Von den 6 untersuchten waren 4 bewohnt und enthielten: Trichosoma contortum Crepl., Filaria tricuspis Fedtsch., Dispharagus anthuris Rud. 


\section{Corvus frugilegus L.}

16 von 47 untersuchten Exemplaren waren von Nematoden frei, allerdings wurde der Oesophagus nie berücksichtigt.

Es beherbergten: Ascaris ensicaudata Rud. 1, Trichosoma resectum Duj. 15, Filaria tricuspis Fedtsch. 3, Dispharagus anthuris 21 Saatkrähen.

Maximalzahlen: Ascaris ensicaudata 1, Trichosoma resectum 43 (8 $\sigma^{x}, 35$ Q), Filaria tricuspis 4 (1 $\sigma^{x}, 3$ ○), Dispharagus anthuris 47 (18 ơ, 29 Q).

10 Individuen von Corvus frugilegus enthielten 2 Arten von Rundwürmern (Trichosoma resectum, Dispharagus anthuris; Filaria tricuspis, Dispharagus anthuris). 1 Individuum enthielt 3 Species zugleich (Trichosoma resectum, Filaria tricuspis und Dispharagus anthuris).

XIII. PICA caudata Boie.

Trichosoma resectum Duj.

XIV. Garrulus glandarius L.

Es wurden im ganzen 76 Exemplare (9 ohne Magen) untersucht, von denen 18 Nematoden enthielten.

Bewohnt von Ascaris ensicaudata Rud. waren 5, von Trichosoma resectum Duj. 2, von Filaria attenuata Rud. 5, von Dispharagus anthuris Rud. 6.

Maximalzahlen: Ascaris ensicaudata ca. 20 ( $\sigma^{\top}$ und $Q$ ), Trichosoma resectum 1, Filaria attenuata 6 १, Dispharagus anthuris 5 Q.

Von 2 Nematodenspecies zugleich bewohnt waren 4 Individuen (Ascaris ensicaudata, Filaria attenuata; Trichosoma resectum, Filaria attenuata; Filaria attenuata, Dispharagus anthuris).

XV. Dryocopus Martius L.

Trichosoma Picorum M. C. V. 
XVI. UPUPA EPOPS L.

Spiroptera truncata Crepl.

XVII. Merula vulgaris Leach.

Filaria tricuspis Fedtsch.

XVIII. TuRdus viscivorus L.

Ascaris ensicaudata Rud.

XIX. Alauda spec.

Ascaris ensicaudata Rud.

XX. Tetrao urogallus L.

Von 47 untersuchten Exemplaren beherbergten 19 keine Rundwürmer.

Es wurden bewohnt von Heterakis inflexa Rud. 21, von Heteralis vesicularis Fröl. 1, von Trichosoma longicolle Rud. 9.

Maximalzahlen: Heterakis inflexa $58\left(27 \sigma^{x}, 31\right.$ @), Heterakis vesicularis 1, Trichosoma longicolle ca. 26 (1 $\mathbf{O}^{\mathbf{T}}$, ca. 25 १).

3 Auerhähne enthielten je 2 Arten (Heterakis inflexa, Trichosoma longicolle), 1 enthielt 3 Arten zugleich (Heterakis inflexa, Heterakis vesicularis, Trichosoma longicolle).

\section{Tetrao tetrix L.}

Von den 10 untersuchten waren 6 frei von Rundwürmern, die andern enthielten Heterakis inflexa Rud. (Maximum $2 \sigma^{\top}$, 8 Q) und Strongylus papillatus v. Linst. in grosser Zahl.

XXII. Tetrao bonasia L. (Russland).

Heterakis inflexa Rud. (3 ๑゙, 5 ९).

XXIII. Perdix saxatilis M. W.

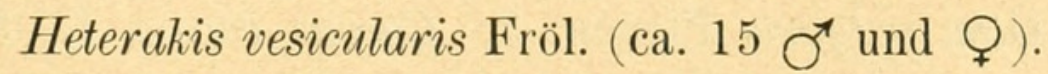


XXIV. Starna CINEREA L.

11 von den 16 untersuchten waren von Nematoden unbewohnt, 4 enthielten Heterakis vesicularis Fröl., 1 Trichosoma longicolle Rud.

XXV. Gallus domesticus Briss.

Heterakis vesicularis Fröl.

XXVI. Phasianus colchicus L.

Im ganzen wurden 10 untersucht, davon waren 7 von Nematoden besetzt, alle diese enthielten Heterakis vesicularis Fröl. (Maximum über 20) und 1 ausserdern Trichosoma longicolle Rud.

XXVII. Phasianus pictus L.

Heterakis vesicularis Fröl. in grosser Zahl.

XXVIII. Crossoptilon mantschuricus Swinhol. (in Gefangenschaft).

Heterakis vesicularis Fröl.

XXIX. Polyplectron bicalcaratum Gray.

Heterakis vesicularis Fröl. (über 20).

XXX. Pavo cristatus L.

Heterakis vesicularis Fröl. (ca. 15).

XXXI. OTIS TARDA L. (zoolog. Garten).

Trichosoma brevicolle Rud.

XXXII. PODICEPS MINOR Gm.

Ascaris spiculigera Rud. (11 $\mathrm{O}^{\top}, 4$ ○).

Der Reichtum an Nematodenspecies ergibt für die untersuchten Vögel folgende Reihenfolge: Accipiter nisus L. 5 Species, Corvus corone L. 5, Corvus frugilegus L. 4, Garrulus glandarius L. 4, Otus vulgaris Flemm. 3, Corvus cornix L. 3, 
Tetrao urogallus L. 3. Folgende enthielten 2 Species: Rhea americana Lam., Tetrao tetrix L., Starna cinerea L., Phasianus colchicus L., die übrigen nur eine Species.

Am regelmässigsten scheinen die Rabenvögel, Raubvögel und Hühner von Nematoden besetzt zu sein, gleichzeitig treten bei ihnen die Rundwürmer in grösster Individuenzahl auf.

Sitz der Nematoden :

\begin{tabular}{|c|c|c|}
\hline Intestinum & 10 & Specie \\
\hline Сœесum & 4 & $»$ \\
\hline Cavum abdominale & 4 & $\gg$ \\
\hline Ventriculus & 3 & $\gg$ \\
\hline Oesophagus & 2 & $»$ \\
\hline Cavum thoracale & 1 & $»$ \\
\hline Musculatur & 1 & » \\
\hline Tunica ventriculi & 1 & » \\
\hline Ingluvies & 1 & $»$ \\
\hline
\end{tabular}

Für folgende Arten wurden neue Wirte gefunden :

Ascaris ensicaudata Rud. in Alauda spec.

Heterakis inflexa Rud. in Tetrao tetrix und Tetrao bonasia.

Heterakis vesicularis Fröl. in Crossoptilon mantschuricus und Polyplectron bicalcaratum.

Strongylus papillatus v. Linst. in Tetrao tetrix.

Trichosoma Picorum M. C. V. in Dryocopus Martius.

Trichosoma resectum Duj. in Pyrrhocorax alpinus, Corvus corone und Pica caudata.

Trichosoma brevicolle Rud. in Otis tarda.

Trichosoma obtusum Rud. in Brachyotus palustris.

Filaria tricuspis Fedtsch. in Merula vulgaris. 


\section{Zur Bestimmung wurde folgende Litteratur benutzt:}

Diesing, C. M. - Systema Helminthum, 18501.

Dujardin, F. - Histoire naturelle des Helminthes, 184.0

Eвerth, J. - Untersuchungen über Nematoden, 1863.

v. Linstow, 0. - Compendium der Helminthologie, 1878 und 1889.

- $\quad$ Archiv für Naturgeschichte, XXXIX, 1873.

- $\quad$ Ibidem, XLI, 187 .

- $\quad$ Ibidem, XLIII, 1877.

- $\quad$ Ibidem, XLV, 1879.

- $\quad$ Ibidem, XLVI, 1880.

- Ibidem, XLVIII, 1882.

- $\quad$ Ibidem, XLIX, 1883.

Ibidem, IV, 1884.

Linton, E. - Notes on avian entozoa. Smithsonian Institution, 1892.

MoLin, R. - Denkschrift der k. Akademie, XIX, 1861.

Schneider, A. - Monographie der Nematoden, 1866.

Stossich, M. - Bolletino della Società Adriatica di Scienze Naturali in Trieste, IX, 1887.

- $\quad$ Societas Historico-Naturalis Croatica, IV, 1889.

- $\quad$ Il Genere Physaloptera Rudolphi. Lavoro Monografico, 1889.

- Societas Historico-Naturalis Croatica, V, 1890.

- $\quad l l$ Genere Trichosoma Rudolphi. Lavoro Monografico, 1890.

- Societas Historico-Naturalis Croatica, VI, 1891.

- $\quad$ Il Genere Dispharagus Dujardin. Lavoro Monografico, 1891.

- Il Genere Angiostomum Dujardin, 1893.

- Bolletino della Società Adriatica di Scienze nalurali in Trieste. XIV, 1893.

- $\quad$ Il Genere Ascaris Linné. Lavoro Monografico, 1896.

- Filaria e Spiroptere. Lavoro Monografico, 1897.

Studer, Th. und Fatio, V. - Katalog der schweizerischen Vögel, 1892.

Anmerkung. - Wärend des Druckes erschien :

Müнıng, P. - Die Helminthen-Fauna der Wirbeltiere Ostpreussens. Arch. f. Naturgesch. I. 1898. 


\section{$2 \mathrm{BHL}$ Biodiversity Heritage Library}

Volz, Walter. 1899. "Statistischer Beitrag zur Kenntnis des Vorkommens von Nematoden in Vögeln." Revue suisse de zoologie 6(1), 189-198. https://doi.org/10.5962/bhl.part.75149.

View This Item Online: https://www.biodiversitylibrary.org/item/39595

DOI: https://doi.org/10.5962/bhl.part.75149

Permalink: https://www.biodiversitylibrary.org/partpdf/75149

\section{Holding Institution}

MBLWHOI Library

\section{Sponsored by}

MBLWHOI Library

\section{Copyright \& Reuse}

Copyright Status: NOT_IN_COPYRIGHT

This document was created from content at the Biodiversity Heritage Library, the world's largest open access digital library for biodiversity literature and archives. Visit BHL at https://www.biodiversitylibrary.org. 